\title{
On the Concept of the National Visualization System for Aerogasdynamic Computations
}

\author{
P. Silvestrov ${ }^{1}$, O. Bessonov ${ }^{2}$, V. Yarmolyuk ${ }^{1}$ \\ ${ }^{1}$ Tactical Missiles Corporation, Korolev, Moscow district, 141080, Russia \\ ${ }^{2}$ Institute for Problems in Mechanics of the Russian Academy of Sciences, \\ Moscow, 119526, Russia \\ pavelsilvestrov@yandex.ru
}

\begin{abstract}
This paper presents the general idea and the first implementation of the new visualization system - an integrated environment for viewing and analyzing results of aerogasdynamic computations and different spatial objects (vehicle surfaces, computational grids). This system is based on the open-source cross-platform development toolkits Qt and VTK. The paper describes basic functions of the visualization system and presents examples of its graphical capabilities for drawing spatial images and two-dimensional plots. Examples of dialogue boxes for controlling the process of visualization are also shown. The system supports main geometric and grid formats, as well as it is partially compatible with the popular commercial visualization system Tecplot (both in data formats and control procedures). The new system has the unified implementation for Windows and Linux operation systems and can be deployed on any modern personal computer.
\end{abstract}

Keywords: gas dynamics, computational aerodynamics, interactive information and simulation system, computer graphics.

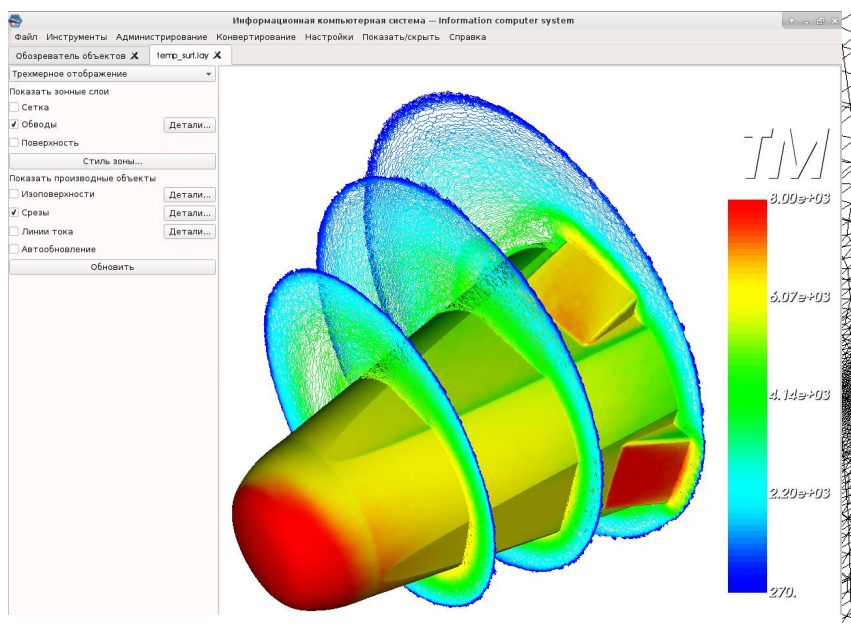

Example of the spatial visualization window showing the distribution of temperature on the surface of a vehicle and in cross-sections

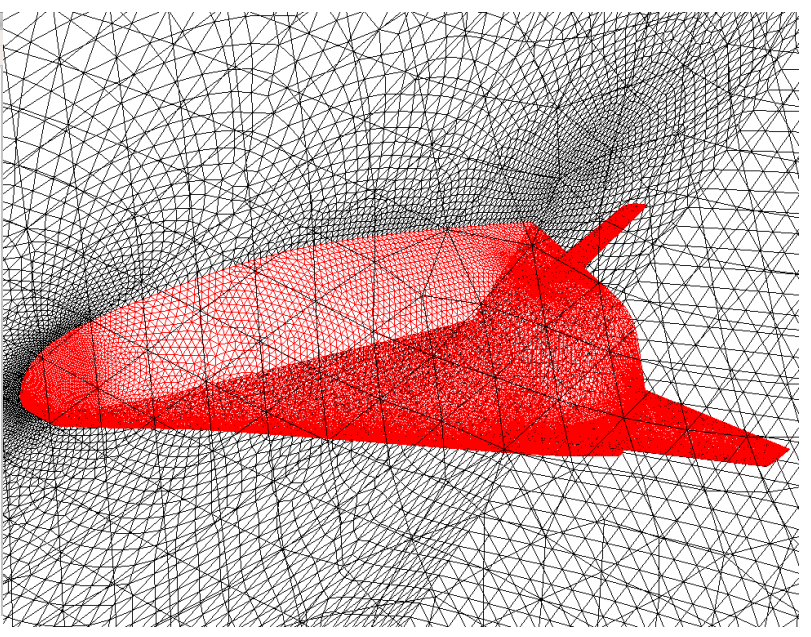

Example of the presentation of a computational grid; zone that corresponds to a surface of a vehicle is highlighted 


\title{
Концепция создания отечественного визуализатора результатов аэротермогазодинамических расчетов
}

\author{
П.В. Сильвестров ${ }^{1}$, О.А. Бессонов ${ }^{2}$, В.Н. Ярмолюк ${ }^{1}$ \\ ${ }^{1}$ Корпорация «Тактическое ракетное вооружение», \\ Россия, Московская обл., Королев, 141080, ул. Ильича, 7 \\ ${ }^{2}$ Институт проблем механики им. А.Ю. Иилинского Российской академии наук, \\ Россия, Москва, 119526, проспект Вернадского, 101-1 \\ pavelsilvestrov@yandex.ru
}

\begin{abstract}
Аннотация
В статье представлена концепция и описывается реализация первого варианта отечественной интерактивной системы визуализации результатов аэротермогазодинамических расчетов, построенной на основе открытых кроссплатформенных средств разработки Qt и VTK. Описаны возможности системы по визуальному представлению пространственных изображений и двумерных графиков, приведены примеры различных способов графического изображения моделируемых объектов и связанных с ними распределений физических величин. Показаны примеры управления визуализацией с помощью диалоговых окон. Представленная система визуализации поддерживает основные геометрические и сеточные форматы, а также обеспечивает частичную совместимость с распространенной коммерческой системой визуализации Tecplot как по форматам графических данных, так и по способу настройки с помощью управляющих файлов. Система имеет унифицированную реализацию для операционных систем Windows и Linux и может быть развернута на любом современном персональном компьютере.
\end{abstract}

Ключевые слова: газовая динамика, вычислительная аэродинамика, интерактивная информационная и моделирующая система, компьютерная графика.

\section{1. Введение}

В процессе разработки новых моделей летательных аппаратов возникает необходимость проведения компьютерных математических имитационных расчетов аэротермодинамических характеристик [1-6]. Такие расчеты позволяют значительно сократить количество наземных и летных испытаний, а также существенно снизить стоимость разработки будущего аппарата. В [7] была представлена интерактивная информационная и моделирующая система (ИИМС), объединяющая вычислительные модули для проведения аэрогазодинамических расчетов, диалоговую графическую оболочку для управления вычислениями и средства информационной поддержки расчетов. Система позволяет проводить весь комплекс работ по моделированию аэродинамических и газодинамических свойств летательного аппарата и его составных частей. В число функций информационно-моделирующей системы входят:

- общее управление всеми этапами вычислений от подготовки входных данных до визуализации и анализа результатов расчетов;

- построение расчетной сетки на основе заранее подготовленной электронной геометрии объекта; 
- численное определение аэродинамических и тепловых нагрузок на поверхность и элементы летательного аппарата;

- моделирование термогазодинамических процессов во внешних и внутренних потоках, учитывая сгорание;

- моделирование высокотемпературных потоков в ударном слое вблизи летательного аппарата и в интегрированном прямоточном двигателе;

- моделирование взаимодействия высокотемпературных газов с теплозащитным экраном спускаемого аппарата;

- моделирование неравновесных химических и фотохимических процессов, а также формирования кластеров и аэрозольных частиц в вытяжных струях двигателя;

- определение сигнатуры струи и моделирование способов подавления естественных и искусственных атмосферных возмущений;

- доступ к набору стандартных тестовых задач, созданных на основе наземных и летных экспериментальных данных;

- доступ к базе данных аэротермодинамических расчетов для типовых аппаратов.

При работе с информационно-моделирующей системой возникает проблема обработки результатов расчетов и их визуального представления. В настоящий момент большинство расчетных модулей, входящих в состав системы, выдают результаты численного моделирования в форматах, предназначенных для графического представления в коммерческом средстве визуализации Tecplot [8]. Данное средство нашло широкое применение в вычислительной гидродинамике и аэродинамике и стало де-факто одним из стандартов для визуализации результатов расчетов. Однако использование коммерческих программных продуктов такого типа в отечественных организациях, занимающихся разработкой перспективных летательных аппаратов, может вызвать различные проблемы лицензионного и иного характера.

Для обеспечения независимости от зарубежного программного обеспечения была поставлена задача определить концепцию отечественной системы визуализации аэрогазодинамических расчетов и реализовать первый вариант такой системы. Одним из требований является обеспечение совместимости с системой визуализации Tecplot по форматам входных данных и, частично, по интерфейсу взаимодействия с пользователем.

В настоящей статье представлен первый вариант системы визуализации, который предлагается внедрить в рамках ИИМС. Система может быть развернута на компьютере, работающем под управлением операционной системы Windows или Linux.

\section{2. Понятийный аппарат}

Визуализация данных - это представление данных в том виде, который обеспечивает наиболее эффективную работу человека по их изучению [9]. Визуализация может использоваться на всех этапах проведения аэрогазодинамических расчетов и включает в себя следующие действия:

- просмотр исходной электронной геометрии объекта;

- просмотр расчетной сетки (этот этап полезен для оценки степени соответствия сетки расчетной области поставленной задаче и визуального контроля характеристик сетки);

- визуализация промежуточных и окончательных результатов расчетов.

В качестве графического отображения процесса визуализации будут рассматриваться трехмерные изображения (например, геометрия поверхности аппарата и распределение тепловых потоков на ней) и двумерные графики (линейные диаграммы). 


\section{3. Функциональный состав системы визуализации}

Внешний вид диалогового интерфейса системы визуализации реализован по подобию интерфейса коммерческой системы Tecplot. В сочетании с совместимостью форматов входных данных это позволяет пользователям, ранее работавшим с системой Tecplot, быстро перейти на новую графическую среду.

На рис. 1 показан пример интерфейса визуализатора вместе с картиной распределения температуры на поверхности моделируемого летательного аппарата и в нескольких сечениях (срезах) расчетной области [10].

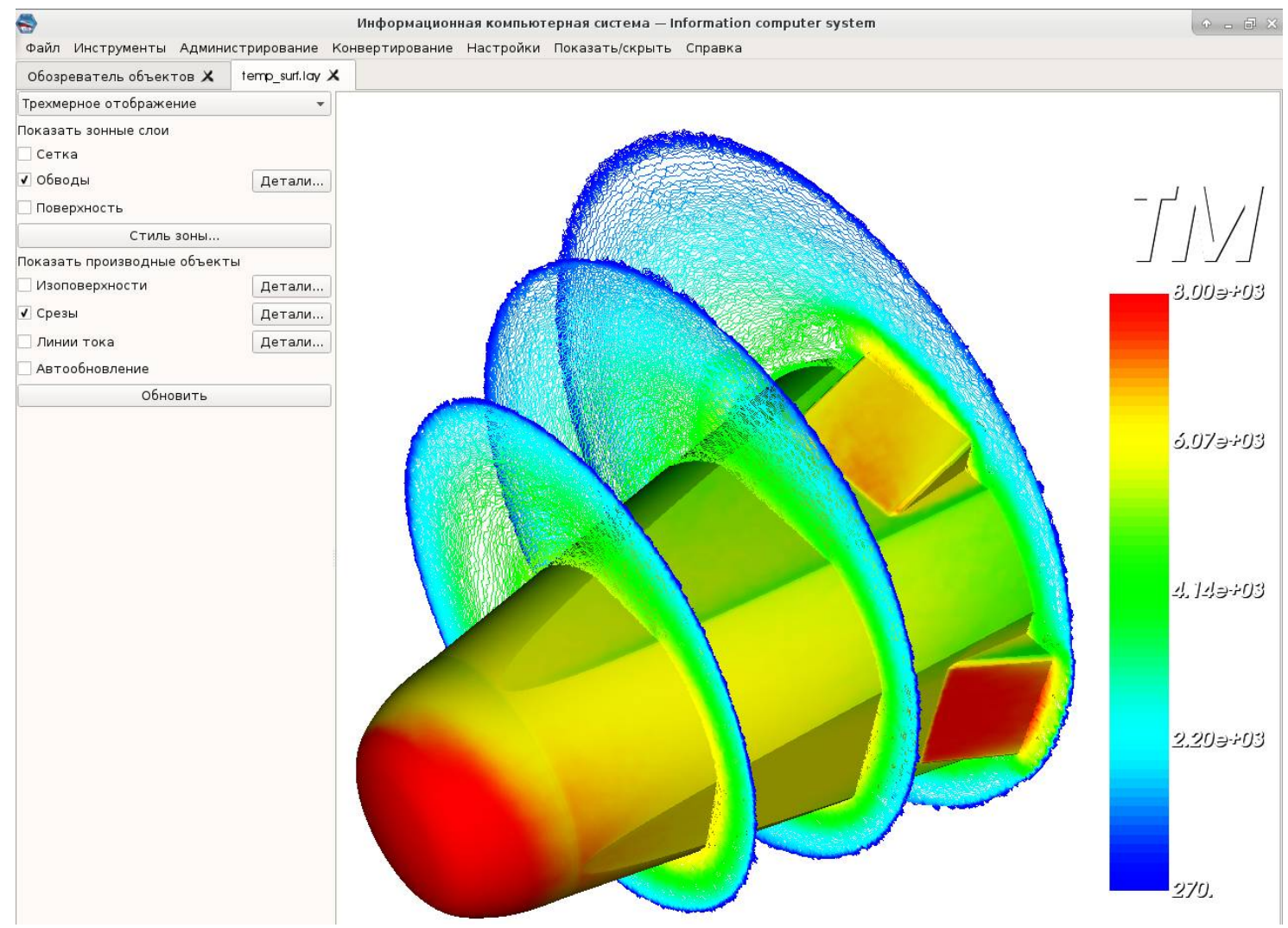

Рис. 1. Пример окна трехмерной визуализации с картиной распределения температуры на поверхности аппарата и срезах расчетной области

Система визуализации поддерживает следующие входные форматы данных:

- стандартные геометрические форматы (*.stl в бинарном и текстовом вариантах);

- стандартные сеточные форматы (*.neu);

- графические форматы распространенных систем визуализации - Tecplot (*.plt, *.dat) и ParaView (*.vtk, *.vtu).

Для настройки сложной визуализации используются управляющие файлы в формате системы Tecplot (*.lay). К настоящему моменту реализована часть возможностей по настройке в таких файлах.

Система визуализации поддерживает два режима вывода данных - трехмерный и двухмерный.

\section{1. Трехмерный режим визуализации}

Трехмерный режим визуализации предназначен для построения и представления пространственных изображений и их фрагментов, в том числе двумерных сечений пространственных распределений физических величин. Ниже приведен список основных типов про- 
странственных изображений, поддерживаемых визуализатором, а также отдельные примеры визуализации:

- контуры изолиний и цветные распределения физических величин в сечениях (срезах) и на поверхностях объектов (рис. 1);

- пространственные изображения поверхностных и объемных расчетных сеток с возможностью скрытия невидимых линий (рис. 2);

- полутоновые изображения поверхностей летательных аппаратов и элементов конструкции (рис. 3);

- картины распределения физических величин в плоских сечениях расчетных областей с возможностью наложения пространственных изображений летательных аппаратов (рис. 4);

- изоповерхности с возможностью вывода на них цветных распределений физических величин (рис. 5);

- траектории и линии тока.



Рис. 2. Визуализация сетки расчетной области для аппарата X-33. Красным цветом выделена зона, соответствующая поверхности летательного аппарата

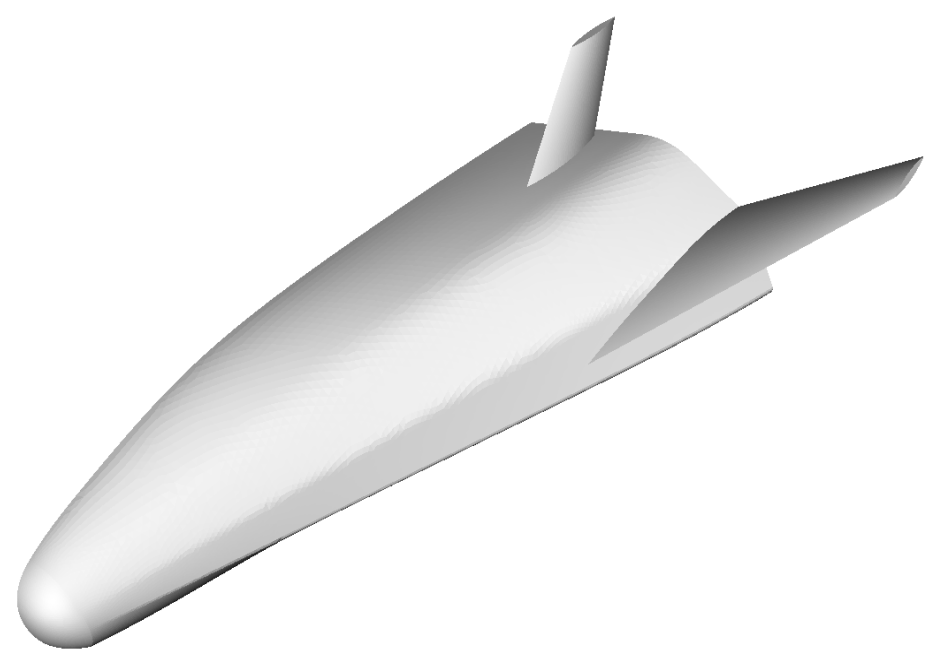

Рис. 3. Визуализация поверхности аппарата X-33 

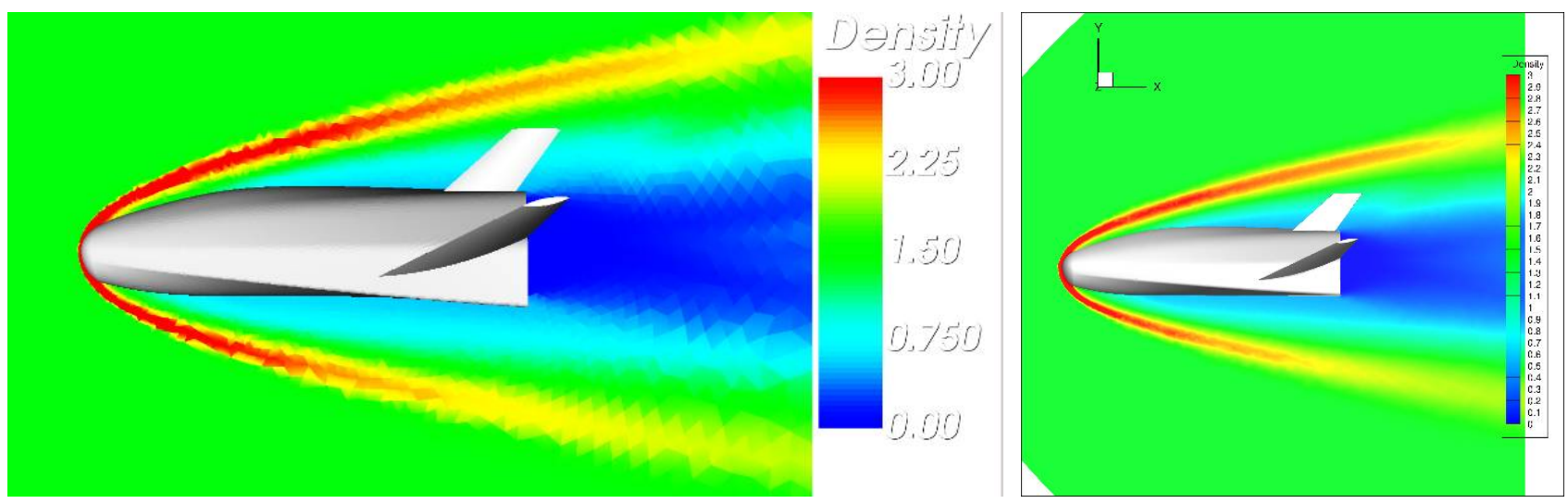

Рис. 4. Картина распределение плотности потока вокруг аппарата X-33 в сечении, близком к плоскости симметрии. Справа для сравнения показан тот же рисунок, выполненный с помощью системы визуализации Tecplot
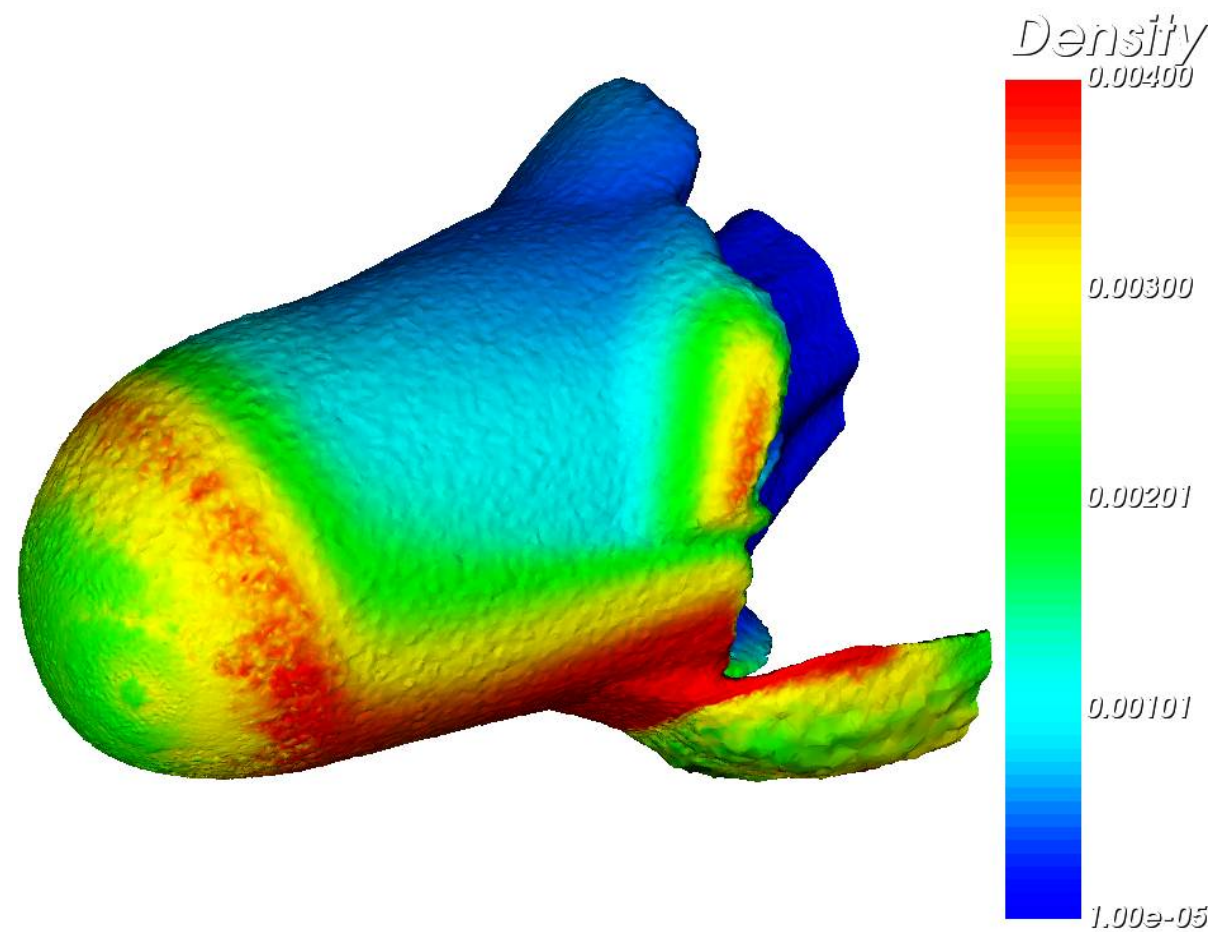

Рис. 5. Пример изображения изоповерхности температуры с выведенным на ней распределением плотности потока

С выведенным изображением возможно проведение различных манипуляций - перемещение по экрану, масштабирование, ориентация и вращение пространственных объектов. Манипуляции производятся с помощью компьютерной мыши.

Настройка изображаемых объектов производится с помощью диалоговых окон с элементами управления. Для указания параметров изображения и степени детализации используются диалоговые окна описания зон, срезов, контуров, изоповерхностей и линий тока.

Диалоговое окно описания зон предназначено для указания следующих параметров и характеристик рисунка с возможностью раздельных настроек в различных зонах (рис. 6):

- перечень зон, которые будут представлены на рисунке;

- указание, для каких зон будут изображены сетки, поверхности, контурные либо цветные распределения, сечения (срезы), изоповерхности и линии тока;

- вид и параметры представления распределения физических величин (контурные изолинии, цветная заливка); 
- цвета сетки, поверхности и внешней линии контура для каждой зоны;

- характер симметрии зоны по отношению к выбранной плоскости.

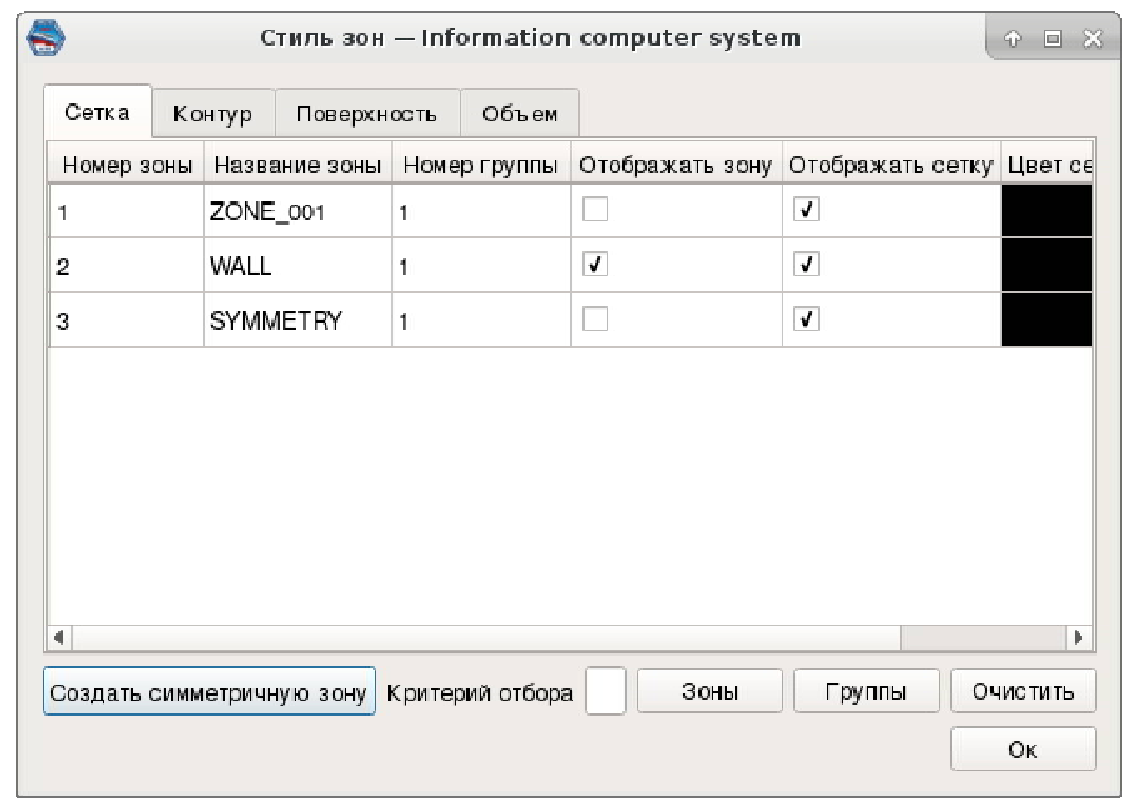

Рис. 6. Пример диалогового окна описания зон

Диалоговое окно описания срезов (сечений расчетной области) предназначено для описания конфигурации и взаимного расположения нескольких срезов, параллельных друг другу (см. рис. 1), и определения способа представления данных на этих срезах (рис. 7). В окне описания срезов пользователь может указать:

- плоскость, указывающую ориентацию срезов;

- точки на оси, перпендикулярной этой плоскости, через которые проходят срезы;

- необходимость изображения поверхности для каждого среза, цвет поверхности;

- необходимость изображения сетки для каждого среза, цвет сетки;

- характер представления распределений физических величин для каждого среза.
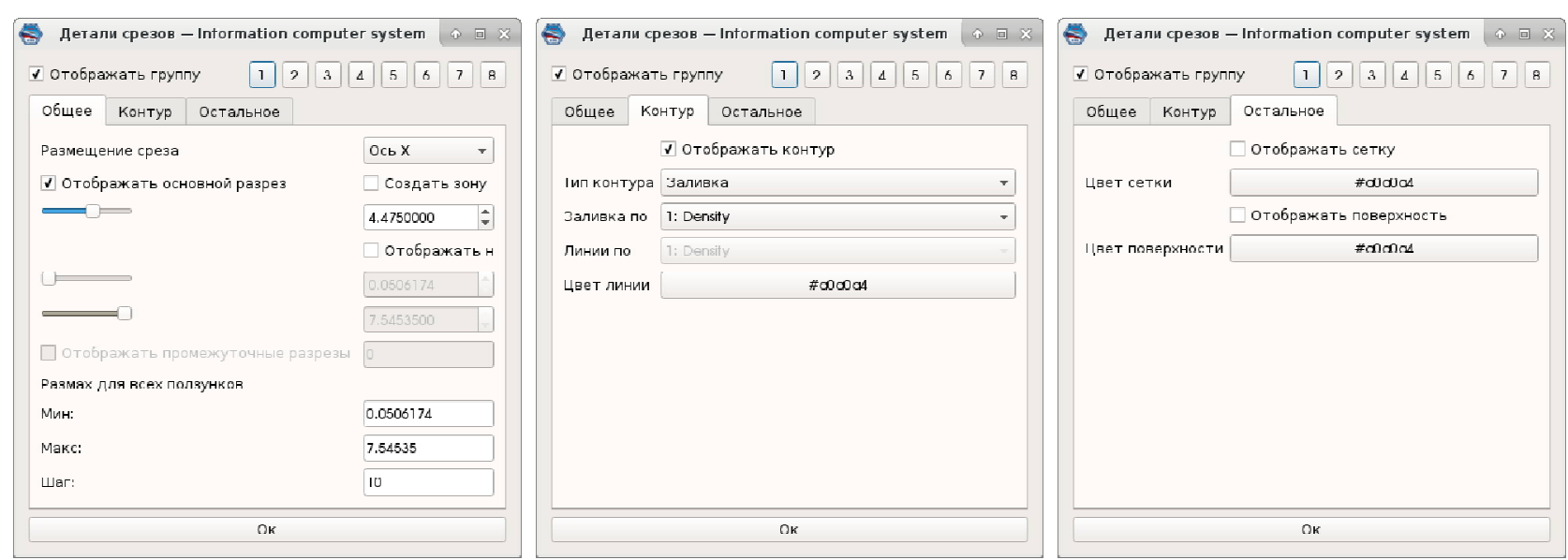

Рис. 7. Пример диалоговых окон, описывающих группу из нескольких срезов

Диалоговое окно описания контуров предназначено для указания способа и параметров представления физических величин на плоскости в виде изолиний либо цветных распределений (рис. 8). В окне указывается два основных набора параметров:

- значения уровней цветового или контурного отображения распределения; 
- подписи на цветовом или контурном отображении.

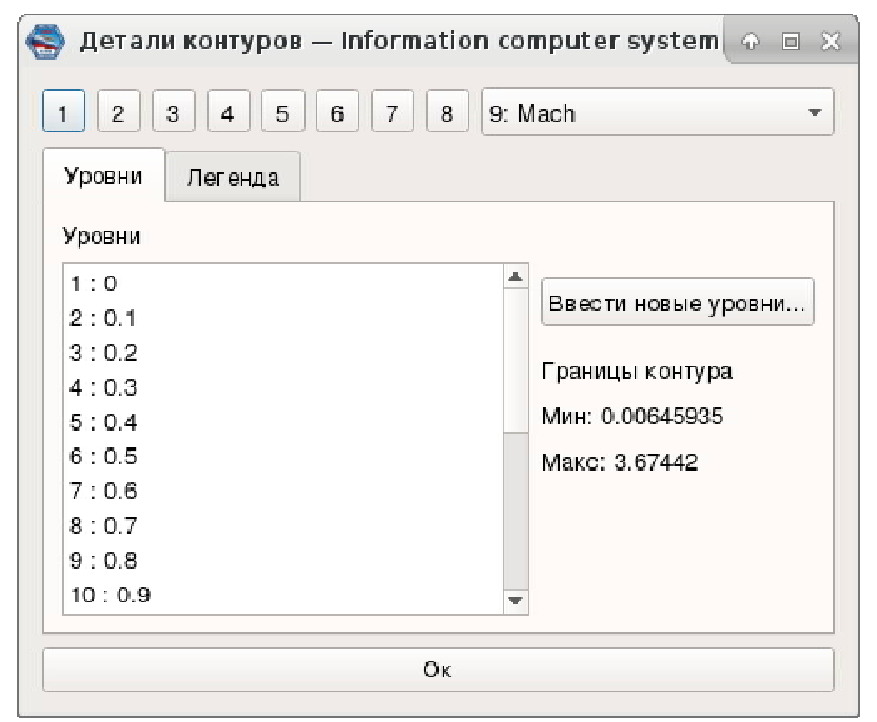

Рис. 8. Пример диалогового окна описания контуров

В диалоговом окне описания изоповерхностей доступен выбор следующих параметров (рис. 9):

- физическая величина, представляемая в виде изоповерхностей;

- значения, для которых изображаются изоповерхности;

- необходимость отрисовки изоповерхностей, цвет изоповерхностей;

- необходимость отображения сетки на изоповерхностях, цвет сетки;

- детали распределения физической величины, выводимого на изоповерхностях.
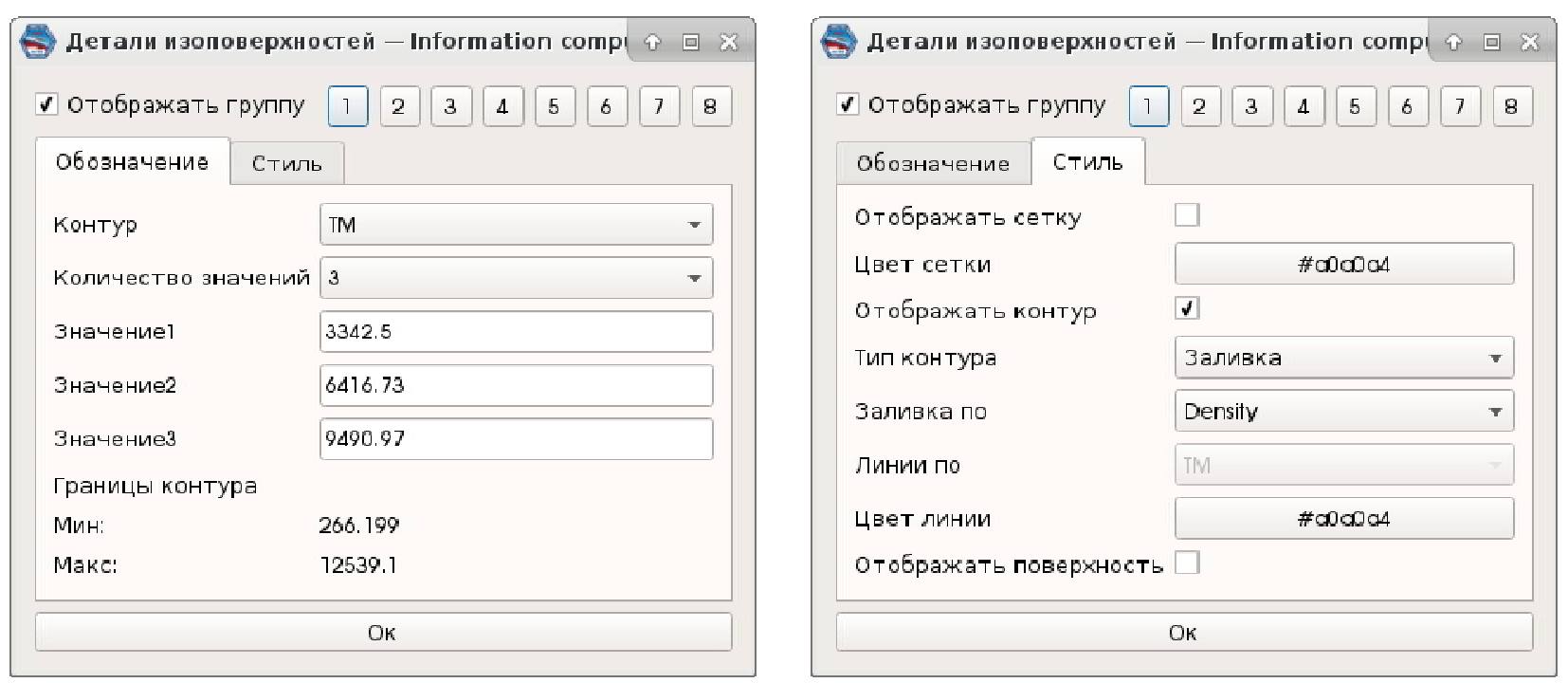

Рис. 9. Пример диалоговых окон, описывающих изоповерхность и выводимое на ней цветное распределение физической величины

Диалоговое окно описания линий тока позволяет указывать характеристики выводимых траекторий, а также производить манипуляции с ними (добавлять новые траектории с указанием начальной точки, либо удалять существующие траектории). 


\section{2. Двухмерный режим визуализации}

Двухмерный режим визуализации предназначен для построения распределения физических величин как функции одной переменной в виде графиков (рис. 10). При построении могут использоваться линии, точки и их сочетания.

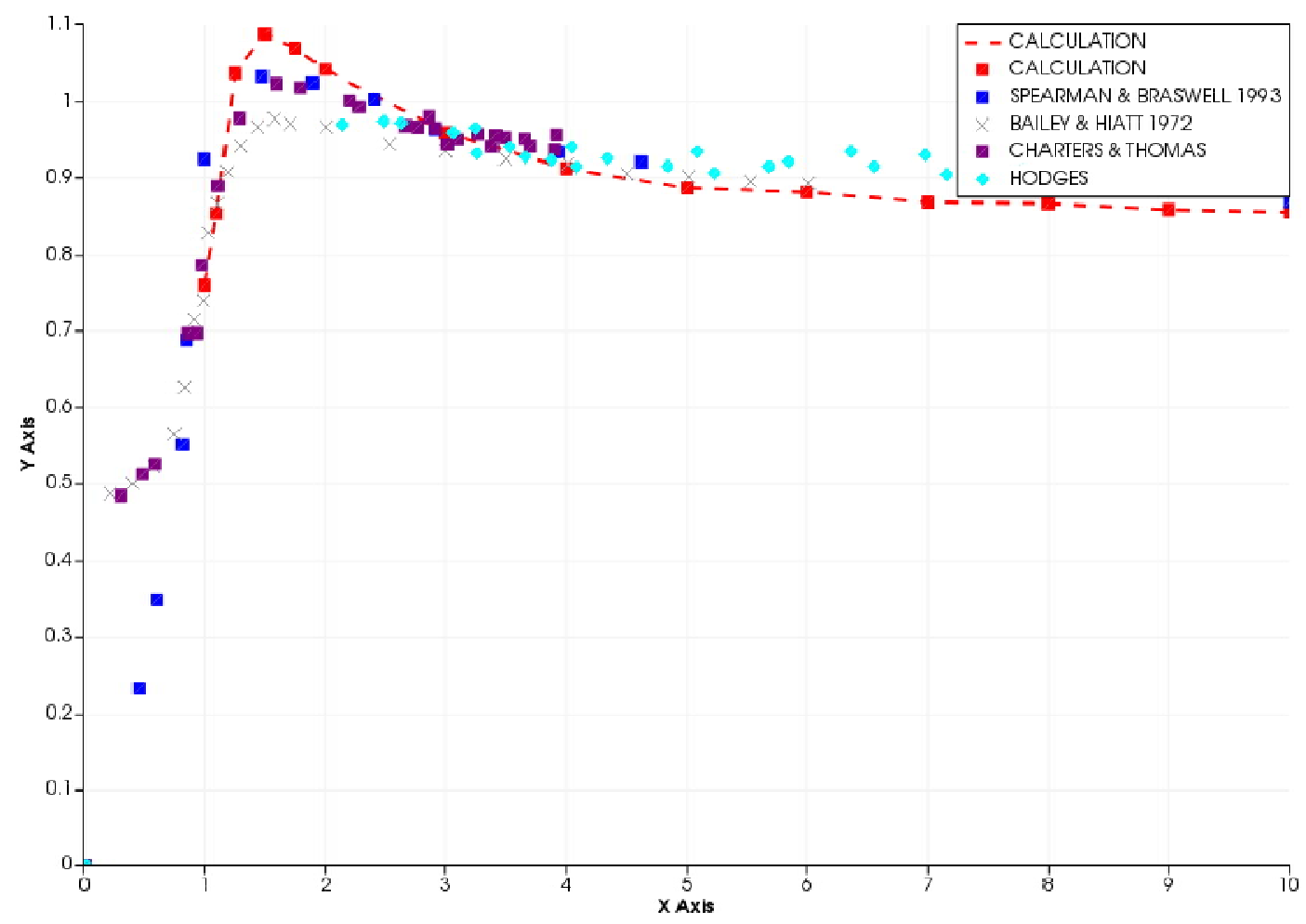

Рис. 10. Двумерный режим. Пример сравнения расчетных и экспериментальных данных

В двумерном режиме управление также производится с помощью диалогового окна (рис. 11), в котором указываются параметры визуализации:

- зависимая и независимая переменные;

- зоны, из которых будут извлечены переменные;

- характер отображения (линии, точки), внешний вид и цвет линий и точек;

- характер вывода подписей (легенды).

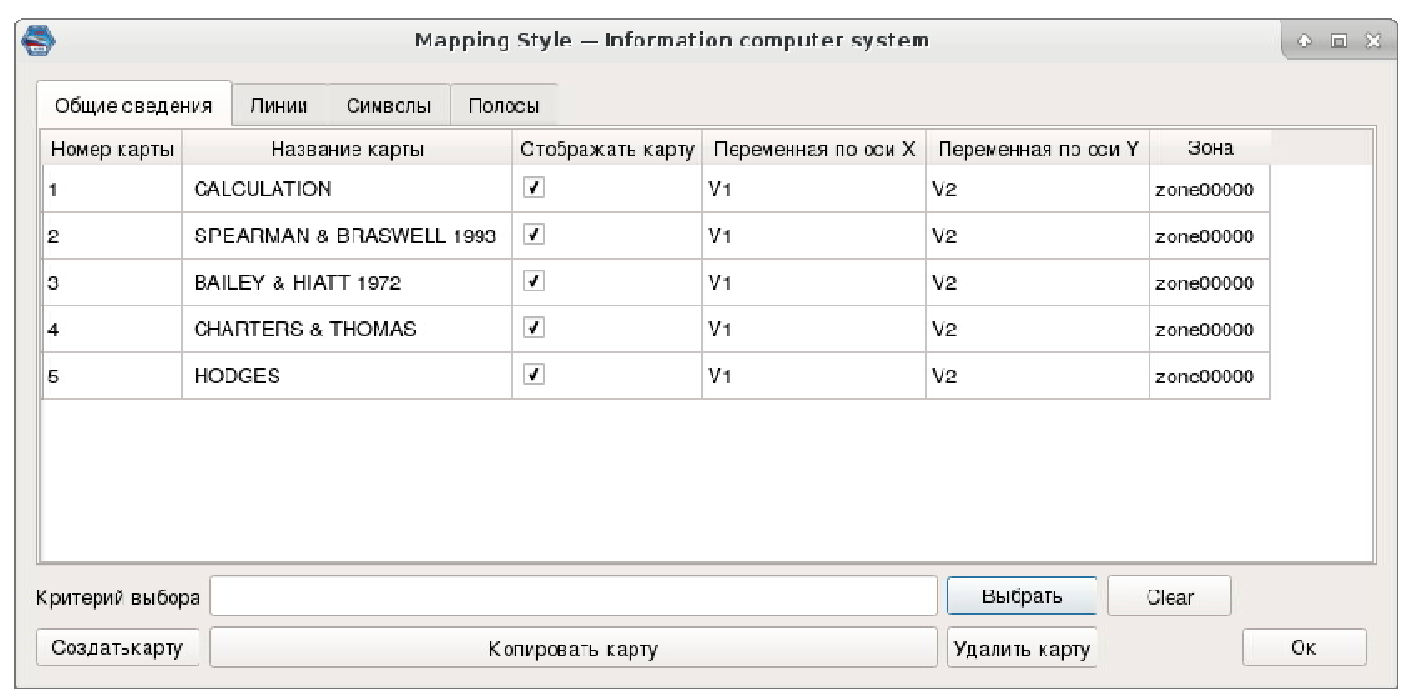

Рис. 11. Пример диалогового окна настройки двухмерной визуализации 
Таким образом, разработанная система визуализации содержит все необходимые элементы и способы графического представления данных, а также инструменты для интерактивного управления процессом просмотра.

\section{4. Реализация и использование системы визуализации}

Система визуализации реализована как составная часть интерактивной информационной и моделирующей системы (ИИМС) [7] с использованием языка программирования $\mathrm{C}++$. Диалоговый графический интерфейс системы реализован с использованием открытой кроссплатформенной среды разработки программного обеспечения Qt $[11,12]$ версии 5.5 . Для вывода графических изображений используется открытая кроссплатформенная программа (библиотека) трёхмерного моделирования, обработки изображений и прикладной визуализации VTK $[13,14]$ версии 7.1.

Новая система была апробирована при решении различных задач для визуализации моделируемой геометрии летательного аппарата [15], расчета траектории [16], пространственного отображения внешнего обтекания [17-21], двумерного отображения [22] и расчета силовой установки [23-25].

\section{5. Заключение}

Разработанная интерактивная система визуализации обеспечивает широкий спектр возможностей для графического представления результатов численного моделирования, а также для просмотра исходной геометрии объекта и расчетной сетки. Система позволяет проводить различные манипуляции с выведенным изображением - перемещение по экрану, масштабирование и вращение. Управление представлением графической информации производится с помощью диалоговых окон.

Система визуализации поддерживает основные геометрические и сеточные форматы и обеспечивает частичную совместимость с коммерческой системой Tecplot по форматам данных и способу управления. Таким образом, представленная система позволяет частично заменить иностранное коммерческое программное обеспечения в рамках импортозамещения. В процессе развития системы добавляется поддержка новых форматов входных данных и расширение функциональных возможностей.

Визуализатор интегрирован в интерактивную информационную и моделирующую систему (ИИМС) и является ее составной частью. Разработанная платформа станет эффективным и удобным инструментом как для проведения аэрогидродинамических расчетов, так и для визуального анализа получаемых результатов.

\section{Благодарности}

Авторы выражают благодарность к.ф.-м.н. А.С. Дикалюку за помощь в реализации отдельных функциональных элементов системы и к.э.н. А.Н. Рыбакову за содействие и поддержку в выполняемой работе.

\section{Литература}

1. Железнякова А.Л., Суржиков С.T. Поле течения около космического аппарата FIRE II под углом атаки// Физико-химическая кинетика в газовой динамике. 2010. Т. 9. http://chemphys.edu.ru/issues/2010-9/articles/139/

2. Surzhikov S.T. Radiative-convective heat transfer of a spherically shaped space vehicle in Carbon dioxide // High Temperature. 2011. Vol. 49. Pp. 92-107

3. Surzhikov S.T. Coupled radiative gasdynamic interaction and non-equilibrium dissociation for large-scale returned space vehicles // Chemical Physics. 2012. Vol. 398. Pp. 56-63 
4. Shang J.S., Surzhikov S.T. Nonequilibrium radiative hypersonic flow simulation // Progress in Aerospace Sciences. 2012. Vol. 53. Pp. 46-65

5. Surzhikov S.T. Radiative-collisional models in non-equilibrium aerothermodynamics of entry probes // J. Heat Transfer. 2012. Vol. 134. 031002

6. Surzhikov S.T. Two-dimensional numerical analysis of flow ionization in the RAM-C-II flight experiment // Russian J. Physical Chemistry B. 2015. Vol. 9. Pp. 69-86

7. Bessonov O.A., Silvestrov P.V. On the concept of the interactive information and simulation system for gas dynamics and multiphysics problems // Journal of Physics: Conference Series. 2017. Vol. 815. 012019

8. Tecplot Home - CFD visualization \& analysis post-processing software. https://www.tecplot.com/

9. Паклин Н. Б., Орешков В. И. Визуализация данных // Бизнес-аналитика. От данных к знаниям. СПб.: Питер, 2013. С. 173-210

10. Kharchenko N.A. Aerothermodynamics calculation of the Expert reentry flight vehicle// Journal of Physics: Conference Series. 2018 (accepted)

11. Qt |Cross-platform software development for embedded \& desktop. https://www.qt.io/

12. Dalheimer M.K. Programming with Qt: Writing portable GUI applications on Unix and Win32. O'Reilly Media, 2002. 499 p.

13. VTK - The Visualization Toolkit. https://www.vtk.org/

14. Schroeder W., Martin K., Lorensen B. The visualization toolkit. Kitware, 2006.

15. Котов М.А. Моделирование поверхности гиперзвукового летательного аппарата // Физико-химическая кинетика в газовой динамике. $2013 . \quad$ Т. 14. http://chemphys.edu.ru/issues/2013-14-4/articles/427/

16. Макеич Г.С., Крюков И.А., Обносов Б.В. Предварительная версия программного модуля расчета динамики полета типовых ГЛА // Физико-химическая кинетика в газовой динамике. 2016. T.17. http://chemphys.edu.ru/issues/2016-17-3/articles/668/

17. Железнякова А.Л. Моделирование аэротермодинамических характеристик виртуального прототипа перспективного сверхзвукового авиалайнера на крейсерском режиме полета // Физико-химическая кинетика в газовой динамике. 2017. Т. 1. http://chemphys.edu.ru/issues/2017-18-1/articles/672/

18. Ermakov M.K., Kryukov I.A. Supercomputer modeling of flow past hypersonic flight vehicles // Journal of Physics: Conference Series. 2017. Vol. 815. 012016

19. Surzhikov S.T. Validation of computational code UST3D by the example of experimental aerodynamic data // Journal of Physics: Conference Series. 2017. Vol. 815. 012023

20. Kotov M.A., Ruleva L.B., Solodovnikov S.I., Surzhikov S.T. Experimental and numerical study of supersonic flow over two blunted wedges // Journal of Physics: Conference Series. 2017. Vol. 815. 012025

21. Yatsukhno D.S. Numerical simulation of the flow over a hypersonic waverider using the method for splitting into physical processes // Journal of Physics: Conference Series. 2017. Vol. 815. 012022

22. Surzhikov S.T. Surface electromagnetic actuator in rarefied hypersonic flow // Journal of Physics: Conference Series. 2017. Vol. 815. 012005

23. Storozhev D.A., Kuratov S.E. Numerical simulation of the kinetics of dissociation and ionization of molecular hydrogen in the Penning discharge plasma with the use of the reduced kinetic model // Journal of Physics: Conference Series. 2017. Vol. 815. 012002 
24. Селезнев Р.К., Суржиков С.Т. Нестационарные газодинамические процессы в прямоугольном канале ГПВРД с периодическим вдувом холодного воздуха //Физико-химическая кинетика в газовой динамике. 2015. T.16. http://chemphys.edu.ru/issues/2015-163/articles/495/

25. Суржиков С.Т. Расчетный анализ данных летного эксперимента по ионизации сжатого слоя при скорости полета 5.34 км/с в разреженной атмосфере // Физико-химическая кинетика в газовой динамике. 2015. T.16. http://chemphys.edu.ru/issues/2015-164/articles/595/

Статья поступила в редакцию 23 января 2018 г. 